\title{
Clonal intratumor heterogeneity of promoter hypermethylation in breast cancer by MS-MLPA
}

\author{
Cathy B Moelans, Jolien S de Groot, Xiaojuan Pan, Elsken van der Wall and \\ Paul J van Diest
}

Department of Pathology, University Medical Center Utrecht, Utrecht, The Netherlands

\begin{abstract}
Intratumor heterogeneity may lead to sampling bias and may present major challenges to personalized medicine and biomarker development. Despite many studies investigating genetic heterogeneity, epigenetic intratumor heterogeneity of promoter hypermethylation has only rarely been examined in breast cancer. To examine clonal intratumor heterogeneity of promotor hypermethylation, we performed methylation-specific multiplex ligation-dependent probe amplification (MS-MLPA) for 24 established tumor-suppressor genes on multiple spatially separated samples obtained from 21 primary breast carcinomas. Multiregion analysis was performed, representing at least two and a maximum of five tumor blocks per patient and four areas per tumor block. Methylation results were heterogeneous at one or more genetic loci between different tumor regions in $95 \%$ of breast carcinomas. The most heterogeneous loci in decreasing frequency were RASSF1A $(62 \%)$, CDKN2B (43\%), APC (38\%), GSTP1 (33\%), CDH13 (24\%), DAPK1 (19\%), and CDKN1B (5\%). Heterogeneity lead to a methylation status change in at least one locus in $65 \%$ of the tumors. For most genes, the relative contribution of between-patients and between-block variability to the total variation in methylation results was similar. Regardless of the gene, contribution of within-block variability was of little importance. In conclusion, although most variation in methylation status is present between individual breast cancers, clonal epigenetic heterogeneity is seen within most primary breast carcinomas, indicating that methylation results from a single random sample may not be representative of the whole tumor.
\end{abstract}

Modern Pathology (2014) 27, 869-874; doi:10.1038/modpathol.2013.207; published online 29 November 2013

Keywords: breast cancer; heterogeneity; hypermethylation; MS-MLPA

Variation within a single tumor, usually denoted intratumor heterogeneity, is a now widely recognized phenomenon characterized by sectors (clonal heterogeneity) and even individual cells (cellular heterogeneity) of different morphology, behavior, and genetic and epigenetic composition. As a result of a combination of cancer stem-like cell differentiation and clonal selection under the influence of the tumor microenvironment, ${ }^{1,2}$ intratumor heterogeneity is a key variable to understand tumor natural history. It may, however, lead to sampling bias and may present major challenges to personalized medicine, predicting disease course and biomarker development.

Molecular evidence suggests that intratumor heterogeneity may contribute to tumor growth through a

Correspondence: Dr CB Moelans, PhD, Department of Pathology, University Medical Center Utrecht, Heidelberglaan 100, PO Box 85500, Utrecht 3508GA, The Netherlands.

E-mail: cmoelans@umcutrecht.nl

Received 29 July 2013; revised 23 September 2013; accepted 23 September 2013; published online 29 November 2013 branched rather than linear pattern of evolution. ${ }^{3}$ Branched tumor evolution underscores the importance of discovering ubiquitous alterations present in all regions of the tumor, ${ }^{4}$ which may lead to superior biomarkers and more efficient targeted therapy.

Most molecular high-throughput and/or genomewide techniques still require relatively large quantities of input DNA, and will thus provide an average picture, usually reflecting the most dominant clones only. Thus, potential heterogeneity will be missed. Although the pooling of large numbers of cells will quench the 'noise' of tumor evolution, minor subpopulations will be left undetected.

During the last decade, many efforts have been made to isolate and compare (epi)genetically distinct subpopulations by either regional macro- or microdissection before analysis and/or by using next-generation sequencing techniques. ${ }^{5,6}$ Depending on the study approach (focused versus genomewide), sampling and the resolution of the analysis method, several studies have revealed either frequent $^{7-10}$ or little $\mathrm{e}^{11-13}$ intratumor heterogeneity. 
Despite many studies investigating genetic heterogeneity, epigenetic intratumor heterogeneity of promoter hypermethylation has only rarely and anecdotally been examined. .,13,14-17 $^{2}$

Aberrant promoter hypermethylation and associated silencing of tumor-suppressor genes occurs early in the course of tumor development and can provide a selective advantage to neoplastic cells. These epigenetic changes are potentially heritable across cell division and are stable over time, but they can also be plastic and allele specific. Nevertheless, it was recently shown that DNA methylation was maintained across all prostatic metastases within the same individual, indicating that these epigenetic alterations can be stably maintained as driver genome alterations fueling cancer initiation and progression. ${ }^{13}$ Aberrant DNA methylation has been observed in all types and stages of cancer, including breast cancer, ${ }^{18-20}$ and measurement of the methylation status of specific genes in tumor tissue or blood can aid early detection of cancer, determine prognosis and predict therapy responses. ${ }^{21}$ A wide range of techniques is available to determine tumor methylation levels, ${ }^{22}$ some of them with very high resolution (such as quantitative multiplex methylation-specific PCR) but often requiring bisulfite conversion and a focused approach, and others with lower resolution (such as MSP and methylation-specific multiplex ligationdependent probe amplification or MS-MLPA). The latter technique does not require bisulfite conversion but instead is restriction enzyme based, and allows a multi-targeted approach on as little as $50 \mathrm{ng}$ of DNA isolated from formalin-fixed paraffinembedded material.

To examine intratumor heterogeneity of promotor hypermethylation, we performed MS-MLPA for 24 established tumor-suppressor genes (26 loci) on multiple spatially separated samples obtained from 21 primary breast carcinomas. Multiregion analysis, representing at least two and a maximum of five tumor blocks per patient and four tumor areas per tumor block, provided evidence of intratumor heterogeneity between different blocks of the same tumor and to a lesser extent between distinct areas within one tumor block.

\section{Materials and methods}

\section{Material}

The present study was performed retrospectively on archival primary breast carcinoma tissue originating from 21 patients who underwent surgery between 2010 and 2011 at the University Medical Center Utrecht. Selected primary ductal breast carcinomas were large enough (on average $3 \mathrm{~cm}$, range 1.2-4.9) to allow sampling of several distinct areas, each measuring $3-6 \mathrm{~mm}$ in diameter, for epigenetic analysis. The mean age was 60 years (range $44-81$ years), mean mitotic activity index was 13 (range 1-50) and all but one of the investigated tumors were estrogen receptor positive. Eight tumors were high grade $(38 \%)$, nine were of intermediate grade $(43 \%)$ and four were low grade (19\%). Fourteen patients showed lymph node metastases (67\%). The study was performed in accordance with the institutional ethical guidelines. Anonymous use of redundant tissue for research purposes is part of the standard treatment agreement with patients in the University Medical Center Utrecht. ${ }^{23}$

\section{Sampling and DNA Extraction}

Four-micrometer sections were cut from each formalin-fixed paraffin-embedded tissue block and stained with hematoxylin and eosin. The hematoxylin and eosin section was used to note pre-invasive lesions, necrosis, and admixed lymphocytic infiltrate and to guide microdissection for DNA extraction. For proteinase K-based DNA extraction, five $10-\mu$ m-thick slides were cut, and tumor areas were microdissected using a scalpel. As illustrated in Figure 1, per patient, one tumor block was subdivided into four non-overlapping areas about equal in size, and DNA was extracted from each of them separately and from the tumor block as a whole. The other tumor blocks from the same patient were also analyzed as a whole. When possible, non-consecutive tumor blocks were used. Areas with necrosis, dense lymphocytic infiltrates, and pre-invasive lesions were intentionally avoided. The DNA concentration and absorbance at 260 and $280 \mathrm{~nm}$ were measured with a spectrophotometer (NanoDrop ND-1000, Thermo Scientific).
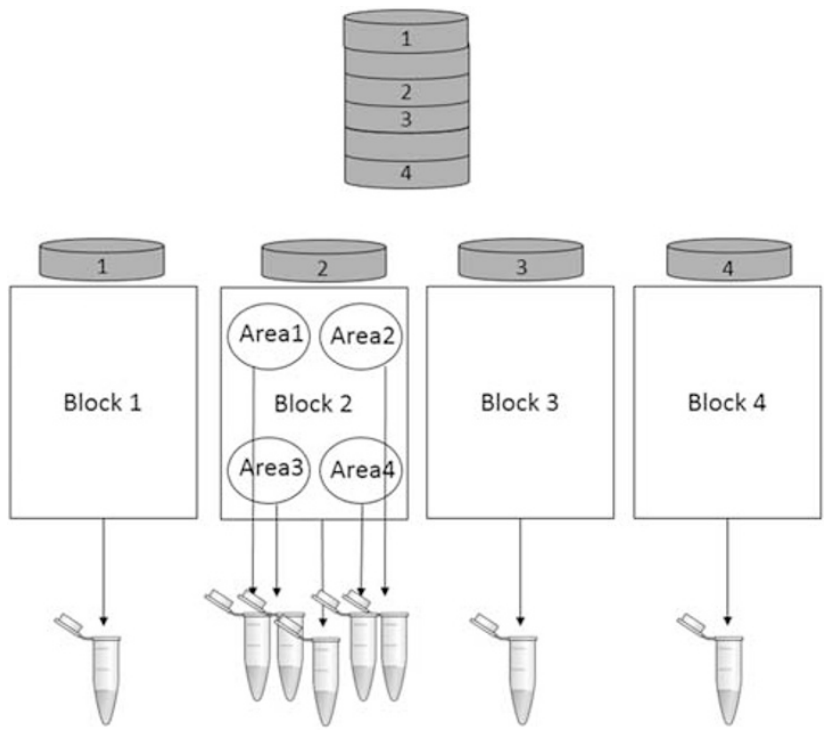

Figure 1 Sampling scheme. From each tumor, two to five (illustrated: four) preferably non-consecutive tumor blocks were analyzed. One of the tumor blocks was further subdivided into four non-overlapping areas about equal in size, and DNA was extracted from each of them separately and from the tumor block as a whole. The other tumor blocks were also analyzed as a whole. 


\section{MS-MLPA}

MS-MLPA was performed according to the manufacturer's protocol using the SALSA MS-MLPA probemix ME001-C1 Tumor suppressor-1 containing 15 control probes and 26 HhaI-sensitive probes against TP73, CASP8, VHL, RARB, MLH1 (2 loci), RASSF1A (2 loci), FHIT, APC, ESR1, CDKN2A/B, DAPK1, PTEN, CD44, GSTP1, ATM, CADM1, CDKN1B, CHFR, BRCA1/2, CDH13, HIC1, and TIMP3. At least $50 \mathrm{ng}$ of DNA was used in each MS-MLPA reaction. All reactions were performed in a Veriti 96 Well Thermo Cycler (Applied Biosystems). A $100 \%$ methylated (sssI methyltransferase treated) control and a negative control (human sperm DNA) were taken along in every MLPA run. Fragment separation was done by capillary electrophoresis on an ABI-3730 capillary sequencer (Applied Biosystems). Peak patterns derived from Genescan Analysis were evaluated using Genemapper software (version 4.1) and Coffalyser software (version 9.4, MRC-Holland). The results were considered reliable if the number of control probes within the normal range (dosage quotient $>0.7$ ) was $\geq 12$. A sample was considered methylated when the dosage quotient was $>0.15$ as before. ${ }^{18}$

\section{Establishment by MLPA of the Criteria for Heterogeneity}

Samples taken from each tumor were assessed for heterogeneity at each locus by calculating the standard deviation and the difference between the maximum and minimum dosage quotient. Dosage quotient heterogeneity was considered present if the standard deviation (s.d.) between different areas and blocks from the same tumor was $>0.07$. This number represents the maximum s.d. seen for any locus without methylation between duplicate or inter-run MS-MLPA measurements. Because the s.d. is known to be higher in highly methylated loci (maximum s.d. of 0.18), the effects of heterogeneity on methylation status calling was also reported.

\section{Statistics}

To evaluate the contribution of within-tumor (between blocks as well as within blocks) and between-tumor variance to the total variation seen in dosage quotient values, a general linear model (for a nested unbalanced ANOVA with a type III error) was used. To evaluate associations of heterogeneity with clinicopathological variables, the $\chi^{2}$ test was used. All statistical calculations were done with IBM SPSS statistics 21.

\section{Results}

Supplementary Table 1 shows MS-MLPA results for all tumor areas and tumor blocks analyzed. Methylation
Table 1 Heterogeneity by MS-MLPA (26 loci) in 21 primary breast carcinomas

\begin{tabular}{|c|c|c|c|c|c|}
\hline Sample & Blocks & $\begin{array}{l}\text { Loci with } \\
\text { s.d. }>0.07\end{array}$ & $\begin{array}{l}\text { Loci with } \\
\text { status } \\
\text { change }\end{array}$ & $B / A$ & Genes involved \\
\hline 1 & 5 & 3 & 2 & B & $\begin{array}{l}\text { CDKN2B, } \\
\text { RASSF1A }\end{array}$ \\
\hline 2 & 5 & 4 & 1 & A & $C D K N 2 B$ \\
\hline 3 & 4 & 3 & 0 & & \\
\hline 4 & 2 & 2 & 1 & B & GSTP1 \\
\hline 5 & 3 & 3 & 1 & A & CDKN2B \\
\hline 6 & 3 & 4 & 2 & $\mathrm{~B}, \mathrm{~A}$ & $C D K N 2 B, C D H 13$ \\
\hline 7 & 4 & 5 & 1 & $\mathrm{~B}, \mathrm{~A}$ & $C D K N 2 B$ \\
\hline 8 & 2 & 2 & 0 & & \\
\hline 9 & 3 & 1 & 0 & & \\
\hline 10 & 3 & 2 & 1 & B & $C D K N 2 B$ \\
\hline 11 & 3 & 3 & 3 & A & $\begin{array}{l}C D K N 2 B, \\
R A S S F 1 A^{\text {a }}\end{array}$ \\
\hline 12 & 3 & 1 & 0 & & \\
\hline 13 & 2 & 3 & 2 & $\mathrm{~B}, \mathrm{~A}$ & $\begin{array}{l}C D K N 2 B \\
C D K N 1 B\end{array}$ \\
\hline 14 & 3 & 1 & 0 & & \\
\hline 15 & 3 & 1 & 0 & & \\
\hline 16 & 3 & 6 & 1 & B & $C D K N 2 B$ \\
\hline 17 & 2 & 3 & 1 & A & GSTP1 \\
\hline 18 & 2 & 0 & 0 & & \\
\hline 19 & 3 & 4 & 4 & A & $\begin{array}{l}A P C, D A P K 1, \\
R A S S F 1 A^{\mathrm{a}}\end{array}$ \\
\hline 20 & 3 & 1 & 0 & & \\
\hline 21 & 3 & 2 & 2 & $\mathrm{~B}, \mathrm{~A}$ & $A P C, C D H 13$ \\
\hline
\end{tabular}

Abbreviations: $\mathrm{B} / \mathrm{A}: \mathrm{B}=$ heterogeneity between different blocks of the same tumor and/or $\mathrm{A}=$ between areas within the same block; MS-MLPA, multiplex ligation-dependent probe amplification. Represented are the number of blocks investigated per tumor, number of loci with heterogeneity, the effects on methylation status calling, the level at which heterogeneity with methylation status change is present $(\mathrm{B} / \mathrm{A})$ and the genes involved.

${ }^{\mathrm{a}}$ Both probes (two different $\mathrm{CpG}$ loci)

in one or more subregions of the tumor was frequently observed for APC (90\%), RASSF1A (81\%), CDH13 (76\%), CDKN2B (57\%), and GSTP1 (52\%). Methylation of DAPK1 (33\%), TP73 (14\%), ESR1 (10\%), and $C D K N 1 B(5 \%)$ was less frequent.

\section{Frequency of Heterogeneity}

Based on the $>0.07$ dosage quotient standard deviation criterion, 20/21 (95\%) breast tumors showed intratumor heterogeneity at one or more of the investigated loci (Table 1, Figure 2). The most heterogeneous loci detected by MS-MLPA in decreasing frequency were RASSF1A (62\%), CDKN2B (43\%), APC (38\%), GSTP1 (33\%), CDH13 (24\%), DAPK1 $(19 \%)$, and $C D K N 1 B(5 \%)$. All other loci did not show intratumor heterogeneity (CASP8, VHL, RARB, MLH1, ATM, FHIT, CDKN2A, PTEN, CD44, IGSF4, CHFR, BRCA1/2, HIC1, TIMP3). This heterogeneity lead to a methylation status change (dosage quotient cutoff of 0.15 ) in at least one locus in 13/20 (65\%) breast tumors. Loci affected by heterogeneity leading to a methylation status change between/within-tumor blocks were CDKN2B (43\% of all 21 samples), RASSF1A (14\%), APC (10\%), GSTP1 (10\%), CDH13 (10\%), DAPK1 (5\%), and CDKN1B (5\%). Heterogeneity in methylation was observed between different 

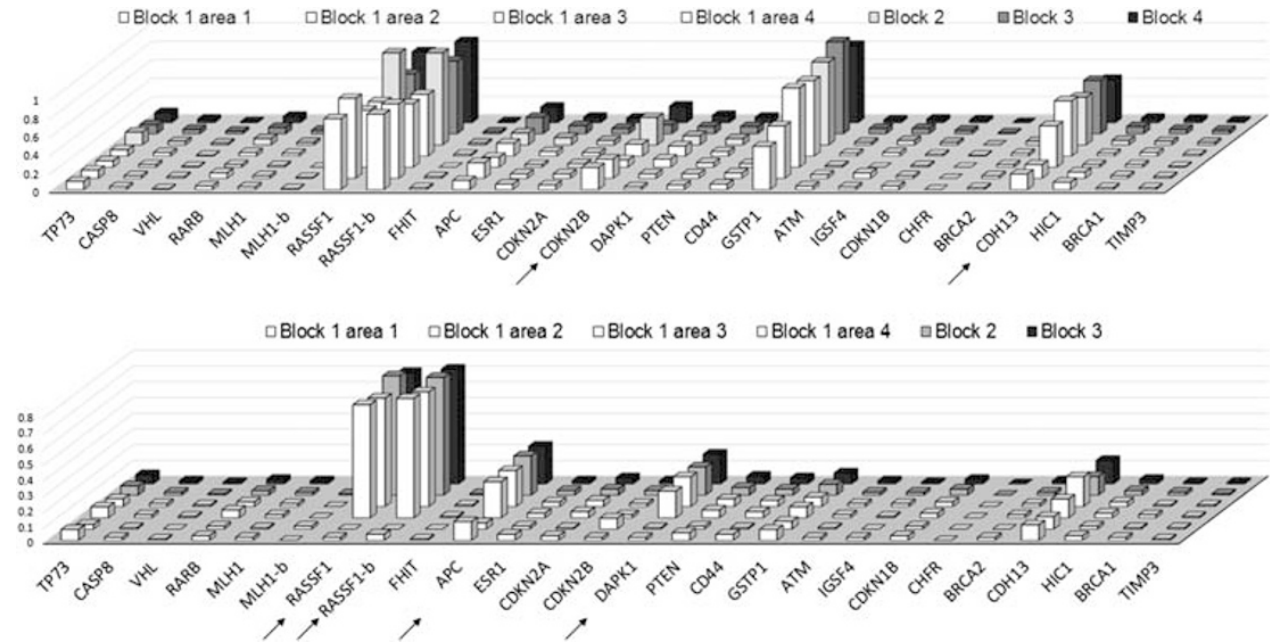

Figure 2 Two samples showing between-block and within-block intratumor heterogeneity in methylation by MS-MLPA. The $x$ axis shows 26 loci, the $y$ axis shows dosage quotient values. Sample 7 (top) shows within-block heterogeneity for CDH13 (not leading to a methylation status change) and between-block heterogeneity for CDKN2B. Sample 19 (bottom) shows a striking within-block heterogeneity for RASSF1A, APC, and DAPK1.

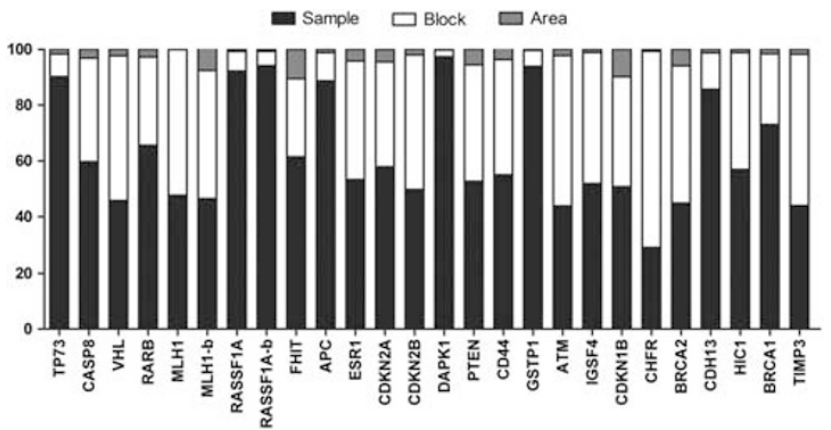

Figure 3 Relative variance contribution of intertumor and intratumor between-block and within-block variability.

blocks of the same tumor as well as between different areas within the same block (Figure 2).

\section{Relative Contribution of Between-Block, Within-Block and Between-Patients (Intertumor) Heterogeneity}

Figure 3 shows the relative contribution of intertumor and intratumor between-block and withinblock variability to the total variance. For most genes, with the exception of TP73, RARB, RASSF1A, FHIT, $A P C, D A P K 1, G S T P 1, C D H 13$, and BRCA1, the relative contribution of intertumor and betweenblock variability to the total variability between observations was similar. For the other genes, intertumor variability was the major cause of heterogeneity contributing up to $97 \%$ to the model. Regardless of the gene, contribution of within-block variability was of little or no importance ( $11 \%$ at most).

\section{Heterogeneity and Clinicopathological Variables}

The degree of heterogeneity, defined by the number of heterogeneous loci per sample, did not correlate with any of the clinicopathological variables (grade, lymph node status, mitotic index, tumor size, age, or HER2 status).

\section{Discussion}

In light of the promising biomarker potential of methylation assessment in breast cancer, this study used regional microdissection of breast tumors to investigate whether MS-MLPA could detect epigenetically distinct subpopulations in geographically distinct tumor sectors (blocks and areas). Methylation results were heterogeneous (at 1 or more loci) between different subregions of the tumor in all but one of the investigated breast carcinomas (95\%). The degree of heterogeneity varied between the examined breast tumors, ranging from one heterogeneous locus $(4 \%)$ up to six heterogeneous loci $(23 \%)$ per tumor. The most heterogeneous loci detected by MS-MLPA in decreasing frequency were RASSF1A (62\%), CDKN2B (43\%), APC (38\%), GSTP1 (33\%), CDH13 (24\%), DAPK1 (19\%), and $C D K N 1 B(5 \%)$. All other loci did not show intratumor heterogeneity. The observed heterogeneity did not always result in a change in methylation status between different regions of the tumor; heterogeneity lead to a methylation status change in at least one locus in as much as $65 \%$ of breast tumors.

Heterogeneity in methylation was observed between different blocks as well as between different areas of one tumor block, but based on a general linear model, contribution of within-block variability to the total dosage quotient variability between and within samples was minimal. For TP73, RARB, RASSF1A, FHIT, APC, DAPK1, GSTP1, CDH13, and $B R C A 1$, intertumor variability was the major cause of overall heterogeneity, contributing up to $97 \%$ to the model. For all other genes, including CDKN2B, 
the relative contribution of intertumor and betweenblock variability to the total variability between observations was similar. These results suggest major interindividual heterogeneity, and indicate far more stability at the within-block level compared with the between-block level.

The results of this study are in line with previous studies in other types of cancer showing high (epi)genetic interindividual heterogeneity and a lower but significant intratumor heterogeneity. 4,7,8,11,13 The marked interindividual heterogeneity challenges 'one-size-fits-all' approaches for cancer management as well as biomarker development and validation, and highlights the need for personalized medicine approaches. The intratumor heterogeneity can lead to sampling bias and thereby hamper accurate prognostication and treatment decisions. Nevertheless, despite intratumor heterogeneity being present, a minority of tumors $(43 \%$ for $C D K N 2 B, 14 \%$ for $R A S S F 1 A$, $10 \%$ for GSTP1 and $C D H 13$, and $5 \%$ for $D A P K 1$ and $C D K N 1 B$ ) shows heterogeneity also leading to a methylation status change, indicating that methylation will still be picked up but at different levels depending on sampling. Thus, if the measurement variable of non-invasive biomarker studies, such as blood or nipple fluid, is 'methylation present or absent' then the sensitivity would not be affected as much, unless cellular heterogeneity is present and more dramatic.

Although MS-MLPA was able to detect heterogeneity at different levels, it may still miss rare or spatially (and temporally) separated subclones because of the inherent lack of resolution compared with, for example, next-generation sequencing techniques. This study therefore focused on clonal heterogeneity instead of cellular heterogeneity and as such eliminates some (but not all) of the 'noise' of tumor evolution, as many of the variants detectable at the level of individual cells eventually fail to clonally expand. Nevertheless, rare subclones not detected by MS-MLPA may have the capacity to become driver events (and vice versa) as environmental selection pressures change during tumor growth and therapeutic intervention. ${ }^{24}$

In summary, we have demonstrated that clonal epigenetic heterogeneity is present within most primary breast carcinomas and can lead to aberrant methylation status calling by MS-MLPA, indicating that analysis of a single random sample may not be representative of the whole tumor, which may hamper epigenetic biomarker discovery and validation. Intertumor heterogeneity was nevertheless more pronounced than intratumor heterogeneity. Although limited intratumor heterogeneity was seen between distinct areas within one tumor block and could lead to a dilution of the overall methylation signal, the heterogeneity between different blocks of the same tumor was significantly larger and is therefore more problematic for clinical purposes.

\section{Disclosure/conflict of interest}

The authors declare no conflict of interest.

\section{References}

1 Almendro V, Fuster G. Heterogeneity of breast cancer: etiology and clinical relevance. Clin Transl Oncol 2011;13:767-773.

2 Campbell LL, Polyak K. Breast tumor heterogeneity: cancer stem cells or clonal evolution? Cell Cycle 2007; 6:2332-2338.

3 Yap TA, Gerlinger M, Futreal PA, et al. Intratumor heterogeneity: seeing the wood for the trees. Sci Transl Med 2012;4:127ps10.

4 Gerlinger M, Rowan AJ, Horswell S, et al. Intratumor heterogeneity and branched evolution revealed by multiregion sequencing. N Engl J Med 2012;366: 883-892.

5 Russnes HG, Navin N, Hicks J, et al. Insight into the heterogeneity of breast cancer through next-generation sequencing. J Clin Invest 2011;121:3810-3818.

6 Shibata D. Cancer. Heterogeneity and tumor history. Science 2012;336:304-305.

7 Benetkiewicz M, Piotrowski A, Díaz De Ståhl T, et al. Chromosome 22 array-CGH profiling of breast cancer delimited minimal common regions of genomic imbalances and revealed frequent intra-tumoral genetic heterogeneity. Int J Oncol 2006;29:935-945.

8 Varley KE, Mutch DG, Edmonston TB, et al. Intratumor heterogeneity of MLH1 promoter methylation revealed by deep single molecule bisulfite sequencing. Nucleic Acids Res 2009;37:4603-4612.

9 Dopierala J, Damato BE, Lake SL, et al. Genetic heterogeneity in uveal melanoma assessed by multiplex ligation-dependent probe amplification. Invest Ophthalmol Vis Sci 2010;51:4898-4905.

10 Navin N, Kendall J, Troge J, et al. Tumour evolution inferred by single-cell sequencing. Nature 2011;472: 90-94.

11 Matsunoki A, Kawakami K, Kotake M, et al. LINE-1 methylation shows little intra-patient heterogeneity in primary and synchronous metastatic colorectal cancer. BMC Cancer 2012;12:574.

12 Trautmann K, Steudel C, Grossmann D, et al. Expression profiling of gastric cancer samples by oligonucleotide microarray analysis reveals low degree of intra-tumor variability. World J Gastroenterol 2005;11: 5993-5996.

13 Aryee MJ, Liu W, Engelmann JC, et al. DNA methylation alterations exhibit intraindividual stability and interindividual heterogeneity in prostate cancer metastases. Sci Transl Med 2013;5:169ra10.

14 Aggerholm A, Guldberg P, Hokland M, et al. Extensive intra- and interindividual heterogeneity of p15INK4B methylation in acute myeloid leukemia. Cancer Res 1999;59:436-441.

15 Bhawal UK, Tsukinoki K, Sasahira T, et al. Methylation and intratumoural heterogeneity of 14-3-3 sigma in oral cancer. Oncol Rep 2007;18:817-824.

16 Korshunova Y, Maloney RK, Lakey N, et al. Massively parallel bisulphite pyrosequencing reveals the molecular complexity of breast cancer-associated cytosine-methylation patterns obtained from tissue and serum DNA. Genome Res 2008;18:19-29. 
17 Rastetter M, Schagdarsurengin U, Lahtz C, et al. Frequent intra-tumoural heterogeneity of promoter hypermethylation in malignant melanoma. Histol Histopathol 2007;22:1005-1015.

18 Moelans CB, Verschuur-Maes AH, van Diest PJ. Frequent promoter hypermethylation of BRCA2, CDH13, MSH6, PAX5, PAX6 and WT1 in ductal carcinoma in situ and invasive breast cancer. J Pathol 2011;225:222-231.

19 Kornegoor R, Moelans CB, Verschuur-Maes AH, et al. Promoter hypermethylation in male breast cancer: analysis by multiplex ligation-dependent probe amplification. Breast Cancer Res 2012;14:R101.

20 Verschuur-Maes AH, de Bruin PC, van Diest PJ. Epigenetic progression of columnar cell lesions of the breast to invasive breast cancer. Breast Cancer Res Treat 2012;136:705-715.

21 Duffy MJ, Napieralski R, Martens JW, et al. EORTC PathoBiology Group. Methylated genes as new cancer biomarkers. Eur J Cancer 2009;45:335-346.

22 Suijkerbuijk KP, Pan X, van der Wall E, et al. Comparison of different promoter methylation assays in breast cancer. Anal Cell Pathol (Amst) 2010;33: 133-141.

23 van Diest PJ. No consent should be needed for using leftover body material for scientific purposes. BMJ 2002;325:648-651.

24 Diaz-Cano SJ. Tumor heterogeneity: mechanisms and bases for a reliable application of molecular marker design. Int J Mol Sci 2012;13:1951-2011.

Supplementary Information accompanies the paper on Modern Pathology website (http://www.nature.com/ modpathol) 